\title{
Soluble Fms-Like Tyrosine Kinase-1 Is A Marker of Endothelial Dysfunction During Sepsis
}

\author{
Marilena Greco ${ }^{\mathrm{a}, \mathrm{b}}$, Claudio Palumbo ${ }^{\mathrm{a}}$, Fernando Sicuro ${ }^{\mathrm{a}}$, \\ Giambattista Lobreglio ${ }^{a}$
}

\begin{abstract}
Background: Sepsis is currently defined as a life-threatening organ dysfunction caused by a deregulated host response to infection. There is increasing evidence that the endothelium plays a crucial and pathogenic role in sepsis. Profound alterations of the endothelium associated with sepsis include increased leucocytes adhesions, shift to a procoagulant state, vasodilatation, altered barrier function with more permeable capillaries and tissue edema. The vascular endothelial growth factor (VEGF) pathway is involved in the control of microvascular permeability and has been involved in the pathogenesis of conditions associated with endothelial barrier disruption such as sepsis. sFlt-1 is a soluble variant of the VEGF receptor (Fms-like tyrosine kinase-1, Flt-1 or VEGFR-1) able to down-regulate the effects of VEGF by decreasing its signaling. We investigated the possible involvement of sFlt-1 as biomarker of endothelial alteration during sepsis, organ dysfunction and death.
\end{abstract}

Methods: Serum levels of s-Flt1 were measured in 170 hospitalized patients (77 with sepsis, confirmed by positive blood culture), and in 18 healthy volunteers. The sequential organ failure assessment (SOFA) score was determined by using biochemical and clinical parameters. In a small number of patients ( 9 individuals), s-Flt1 concentration was evaluated after negativization of the blood culture.

Results: Serum level of s-Flt1 was significantly higher in septic patients than blood culture-negative patients $(277.7 \pm 52.7$ and $133.4 \pm$ $12.4 \mathrm{pg} / \mathrm{mL}$, respectively, $\mathrm{P}=0.0088$ ), both groups of patients had significantly higher concentration of sFlt-1 than healthy individuals (78.9 $\pm 2.5 \mathrm{pg} / \mathrm{mL}$ ). Among sepsis cases, $68 \%$ was caused by Gram-negative bacteria, $27 \%$ by Gram-positive bacteria and $8 \%$ by Candida species. Serum level of s-Flt1 showed a significant difference between Gramnegative $(274.1 \mathrm{pg} / \mathrm{mL})$ and Gram-positive $(145.7 \mathrm{pg} / \mathrm{mL})$ sepsis. SOFA score (evaluated in 20 patients with sFlt-1 $>190 \mathrm{pg} / \mathrm{mL}$ ) showed a positive trend of correlation with the increasing sFlt-1 level. After blood culture negativization, serum level of sFlt-1 decreased (37\%).

Manuscript submitted June 5, 2018, accepted June 28, 2018

${ }^{a}$ Clinical Pathology and Microbiology Laboratory, Vito Fazzi General Hospital ASL-Lecce, Lecce, Italy

${ }^{b}$ Corresponding Author: Marilena Greco, Clinical Pathology and Microbiology Laboratory, Piazza Muratore, Vito Fazzi General Hospital, ASL-Lecce, Lecce, Italy. Email: grecomarilena@gmail.com

doi: https://doi.org/10.14740/jocmr3505w
Conclusion: Our findings confirm, in a larger population of patients with sepsis, recent evidences that sFlt-1 levels are higher in patients with complicated-sepsis that evolve to septic shock and suggest that sFlt-1 could be a useful biomarker for sepsis severity. An anti-VEGF effect mediated by sFlt-1 could be hypothesized as salvage compensatory mechanism activated in response to sepsis.

Keywords: Sepsis; Endothelium dysfunction; sFlt-1; SOFA score

\section{Introduction}

Vascular endothelial cells are critically involved in the pathogenesis of sepsis-induced organ failure given their participation in coagulation, increased permeability, vasodilatation, vascular barrier and leukocytes activation and trafficking. Because of their ubiquitous distribution throughout all vascular beds, endothelial cells participate in early and delayed inflammatory responses in sepsis $[1,2]$. During the early phases of sepsis, endothelial cells are directly activated by microbial factors such as lipopolysaccharide (LPS) and bacterial lipoproteins in a permeable, pro-inflammatory and pro-thrombotic phenotype. The activated endothelium is critical for vascular remodeling and repair, leukocytes trafficking and eradication of pathogens.

Subsequently, endogenous inflammatory agonists released by activated leukocytes and endothelial cells further promote endothelial dysfunction, either on their own or in synergy with circulating microbial molecules (pro-inflammatory cytokines promote self-reinforcing increase of innate immune cells, adhesion molecules and chemokines expression, induction of complement and fibrinogen, determine NEToses [3], cause release of inflammatory microparticles [4], up-regulate tissue factor expression by blood monocytes and immuno-thrombosis [5], disseminated intravascular coagulation results in impaired microvascular function and organ injury [6]).

When the response exceeds a certain threshold, systemic injury occurs, and the endothelial response becomes detrimental, uncontrolled, persistent and widespread.

Many circulating biomarkers have been proposed in literature (see [7] for review), including mediators of vasomotor tone and permeability (as VEGF, sFlt-1).

VEGF is a key regulator of angiogenesis, promoting endothelial cell survival, growth and migration [8]. However, it also plays a crucial role in the disruption of endothelium dur- 
ing inflammation, promoting vasodilatation and permeability. Under quiescence condition VEGF signaling plays a critical role in homeostasis. VEGF binds to two Fms-like tyrosine kinase receptors on endothelial cells, VEGFR1 or Flt-1 and VEGFR2 or Flk-1. During activated state (sepsis), circulating levels of VEGF are increased. Elevated VEGF signaling, in turn, leads to increased vascular leak, leukocyte adhesion/trafficking and clot formation. VEGF binds also to a soluble form of the receptor of VEGF (soluble VEGFR1 or sFlt-1). This soluble variant is a truncated protein lacking the tyrosine-kinase domain responsible of intracellular signal transduction, thus acting as a competitive inhibitor of VEGF signaling in endothelial cells. Sepsis-mediated induction of sFlt-1 may represent a critical component of the host anti-inflammatory response. Previous studies have investigated VEGF and sFlt-1 involvement in sepsis and disease severity [9-17] yielding conflicting results if the degree of elevation correlates with the state of disease. sFLT-1 has been recently suggested as a marker for abnormal vascular remodeling state, e.g., preeclampsia [18].

In the present study, we investigated correlation of sFlt-1 levels in sepsis, possibly as a marker of endothelial dysfunction and its correlation with sepsis-related sequential organ failure assessment (SOFA) score including in a group of patients after sepsis resolution.

\section{Materials and Methods}

\section{Patients' population}

The present study has been conducted on 170 consecutive patients hospitalized in different medical and surgical wards of the Vito Fazzi Hospital of Lecce (Italy) for whom blood culture was requested based on different clinical suspicions, and in 18 (not hospitalized) healthy volunteers. Informed written consent was obtained from all patients or, if unobtainable, their relatives' consent was attained as designed by ethic committee. For a subgroup of 9 individuals, serum sample was obtained also after negativization of blood culture.

\section{sFlt-1 assay in serum}

Serum levels of sFlt-1 were determined by fully automated ElectroChemiLuminescence Immuno Asssay (ECLIA) on the immunoanalyzer Elecsys 2010®, Modular Analytics E170, according to manufacturer recommendations (Roche Diagnostic $\mathrm{GmbH}$ ) and expressed in $\mathrm{pg} / \mathrm{mL}$. All sera were collected at the time of blood culture and stored at $-20^{\circ} \mathrm{C}$ until sFlt- 1 assay.

\section{Blood culture}

Blood cultures were performed in patients with clinical symptoms of blood stream infections prior the administration of antimicrobial therapy. For each patient, at least two bottle sets were used for each septic episode; approximately $10 \mathrm{~mL}$ of blood was inoculated in the aerobic and anaerobic bottle
(BACT/ALERT Culture Media, Biomerieux, Marcy-l'Etoile, France); the bottles were entered in the BACT/ALERT 3D System for the incubation and measure of the color change in response to shift in $\mathrm{pH}$ as a result of rising of $\mathrm{CO}_{2}$ levels produced by microorganisms. In positive samples, bacteria and yeasts were identified on the Vitek 2 system (Biomerieux, Marcy-l'Etoile, France), according to manufacturer directions.

\section{Organ dysfunction}

The SOFA score was used to quantify organ dysfunction. The SOFA score, a mortality prediction score, is based on the degree of dysfunction of six organ systems: respiration score is assessed by fraction of inspired oxygen and $\mathrm{PaO}_{2}$, coagulation score by platelets count, liver score by total bilirubin, neurological by Glasgow Coma Scale, cardiovascular score by mean of arterial pressure and use of vasopressors, renal score by serum creatinine or urine output. To each organ, system is assigned a point value from 0 (normal) to 4 (high degree of dysfunction/failure). The SOFA score ranges from 0 to 24; higher value indicates greater organ failure and worst prognosis [19].

\section{Statistical analysis}

Data comparison was performed by Student's $t$-test; statistical significance of difference between analyzed groups of patients was based on $\mathrm{p}$ value $(<0.05)$; Chi-squared test was used to evaluate significance of parameters associated to a clinical condition in different groups. Correlation trend was determined by Chi-squared test for trend. Statistical analyses were performed by MedCalc v18.1.2 statistical software.

\section{Results}

\section{Patients' population}

The population of 170 hospitalized patients analyzed in the present study was subgrouped according to blood culture positivity and according to patient's ward of provenience. We obtained 77 individuals with sepsis (as confirmed by a positive blood culture) and 93 negative, of which only 66 has been used as sepsis-negative controls, while a subgroup of 27 (of the 93 negatives: including 14 surgical patients and 13 new borns) was separately analyzed due to a potential endothelial modification after surgery or after birth, i.e., not baseline condition for sFlt-1 assay. In a small number of patients (nine individuals), s-Flt1 concentration was evaluated after negativization of the blood culture. Not hospitalized individuals (18 healthy volunteers) were used as negative controls (Table 1).

\section{sFlt-1 in sepsis patients}

Serum level of s-Flt1 was significantly higher in septic patients than sepsis-negative patients with average values of $277.7 \pm$ 
Table 1. Patients Population and Percentages of Microganisms Identified in Blood Cultures

\begin{tabular}{lllllll} 
& Healthy controls & No-sepsis controls & Sepsis & New borns & Surgery & Post-sepsis recovery \\
\hline N & 18 & 66 & 77 & 13 & 14 & 9 \\
Age range (years) & $24-65$ & $6-88$ & $20-96$ & $0-28$ days & $6-89$ & $33-89$ \\
Gram-pos. & - & - & $27 \%$ & - & - & $40 \%$ \\
Gram-neg. & - & - & $68 \%$ & - & - & $50 \%$ \\
Candida & - & - & $8 \%$ & - & - & $10 \%$ \\
\hline
\end{tabular}

52.7 and $133.4 \pm 12.4 \mathrm{pg} / \mathrm{mL}$, respectively, and $\mathrm{P}=0.0088$ (Table 2, Fig. 1). Both groups of patients had significantly higher concentration of sFlt-1 than healthy individuals $(78.9 \pm 2.5 \mathrm{pg} /$ $\mathrm{mL}, \mathrm{P}<0.0001)$. However, healthy individuals fit in a different condition compared to hospitalized patients and for this reason, in our study we referred to sepsis-negative hospitalized patients as control group in order to be able to compare patients in similar clinical conditions, besides sepsis.

Among sepsis-positive patients, 68\% were infected by Gram-negative bacteria, 27\% by Gram-positive bacteria and $8 \%$ of patients had Candida positive blood culture (Table 1). Serum level of s-Flt1 (Fig. 2) showed a significant difference between Gram-negative (274.1 pg/mL) and Gram-positive (145.7 pg/ $\mathrm{mL})$ sepsis $(\mathrm{P}=0.021)$. Patients with Candida infection showed an intermediate level of s-Flt1 $(176.5 \mathrm{pg} / \mathrm{mL})$ and no significant difference with Gram-negative and Gram-positive sepsis.

\section{sFlt-1 in surgery and new-born patients}

We separately analyzed sepsis-negative patients from surgery ward as well as new-born patients since they showed an unexpected higher average serum level of s-Flt1 and high interindividual variability (Table 2, Fig. 1). We associated this finding to a potential endothelial modification after surgery or after birth and we excluded them from the group of sepsis-negative controls, although difference between the two subgroups was not statistically significant.

\section{sFlt-1 and sequential organ failure assessment}

Sequential organ failure assessment was evaluated in 20 pa- tients with sepsis whose serum levels of sFlt-1 were higher than $190 \mathrm{pg} / \mathrm{mL}$ (this limit was established considering sFlt1 levels over the upper limit of the $95 \%$ confidence interval (CI) of median value calculated for sepsis patients, Table 2). We observed a positive linear trend between sFlt-1 and SOFA score (Fig. 3a), with significant Chi-squared test for trend $(\mathrm{P}<$ 0.0001). Moreover, patients who survived to sepsis have been differentiated from patients deceased in consequences of the sepsis, and we found that worst clinical condition which determined the death of the patients were associated with higher serum levels of sFlt-1 and SOFA score (Fig. 3b) compared to alive patients $(\mathrm{P}<0.05$ by $\mathrm{Chi}$-squared test $)$.

\section{sFlt-1 after sepsis resolution}

Serum levels of sFlt-1 were measured in nine patients after negativization of blood culture and we found that reduction of sFlt-1 was about $37 \%$. In Gram- negative infections, the percentage of decrease was more evident compared to Grampositive infections. Klebsiella pneumoniae and Pseudomonas aeruginosa infections showed higher range of pre- and postsepsis variation for serum level of sFlt-1 (Fig. 4).

\section{Discussion}

Functional status of the vascular endothelium is of key importance in the maintenance of vasomotor tone, leukocytes trafficking, permeability and hemostasis. In the presence of an infectious disease, endothelial activation/dysfunction can lead to a lesion in microcirculation which can potentially compromise the function of multiple organs. Activation and

Table 2. sFlt-1 Serum Levels Observed in the Analyzed Groups of Patients and Statistical Summary of Results

\begin{tabular}{llllll}
\hline & Healthy controls & No-sepsis controls & Sepsis & New borns & Surgery \\
\hline N & 18 & 66 & 77 & 13 & 14 \\
sFlt-1, mean (pg/mL) & 78.9 & 133.4 & 277.7 & 592.1 & 233.2 \\
SEM & 2.5 & 12.3 & 52.4 & 233.1 & 62.3 \\
95\% CI (mean) & $73.707-84.106$ & $108.933-157.932$ & $173.425-382.030$ & $84.136-1,100.066$ & $98.558-367.811$ \\
sFlt-1, median (pg/mL) & 78.2 & 103.0 & 165.5 & 204.6 & 123.3 \\
95\% CI (median) & $73.773-86.353$ & $94.988-119.330$ & $148.328-191.607$ & $164.783-653.927$ & $93.468-387.771$ \\
5 - 95 percentiles & $58.088-95.328$ & $74.194-359.020$ & $85.572-794.290$ & $122.295-2,836.325$ & $79.710-790.900$ \\
\hline
\end{tabular}

SEM: standard error of the mean; $\mathrm{Cl}$ : confidence interval. 


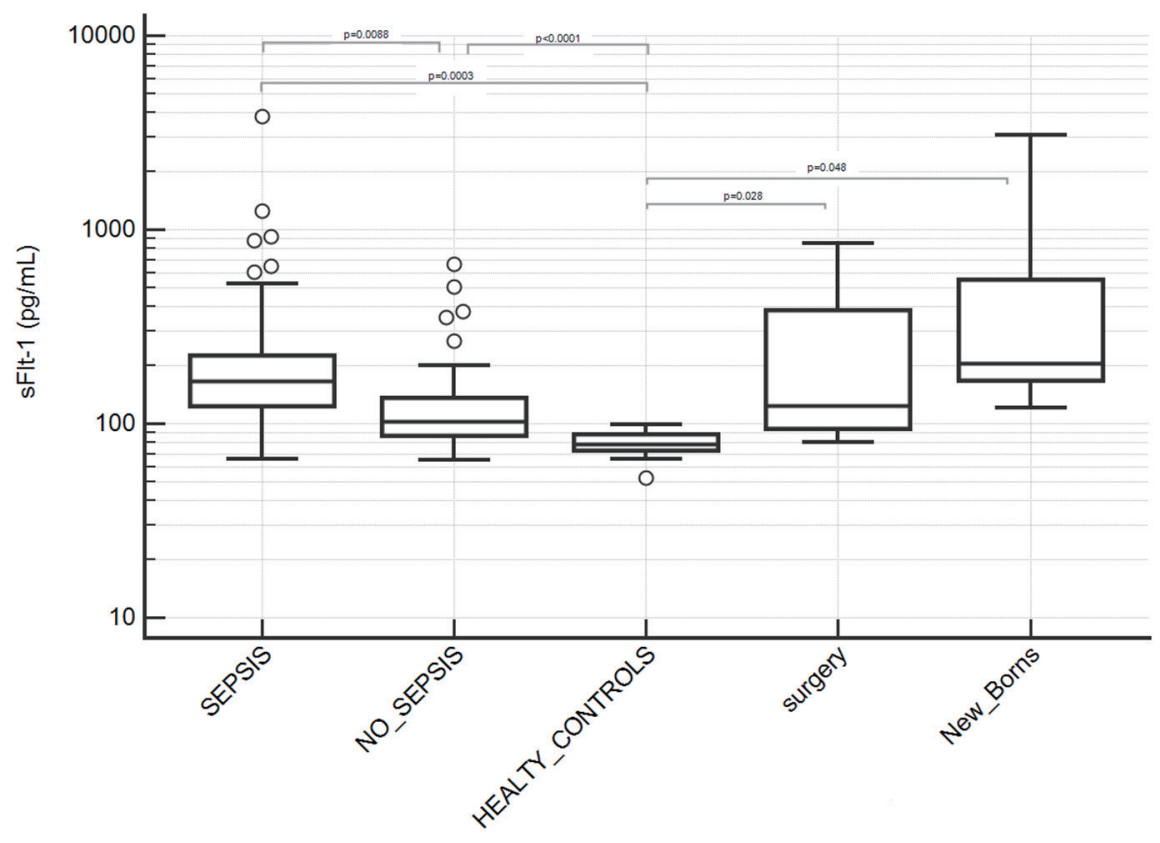

Figure 1. Serum levels of sFlt-1 in patients groups. Septic patients showed significantly higher levels of sFIt-1 compared to sepsis-negative patients and healthy controls ( $P$ values indicated in figure; where not indicated, difference was not statistically significant). Two subgroups of sepsis-negative patients who underwent surgery or new borns were separately analyzed due to a potential not baseline condition for sFlt-1 assay after surgery or after birth; they showed a statistically significant difference with healthy controls but not with sepsis-positive nor -negative patients. Results are showed in semilogarithmic plot.

dysfunction of the endothelium is characterized by increased permeability, vasodilatation, recruitment of leucocytes and a shift to pro-coagulant state. Hence, infections compromise the function of heart, liver, gut, kidney, lung and brain leading to hypotension and myocardial dysfunction, microvascular leak, thrombocytopenia with or without disseminated intravascular coagulation, acute respiratory distress syndrome, acute kidney injury and brain injury. Biochemical markers and clini-

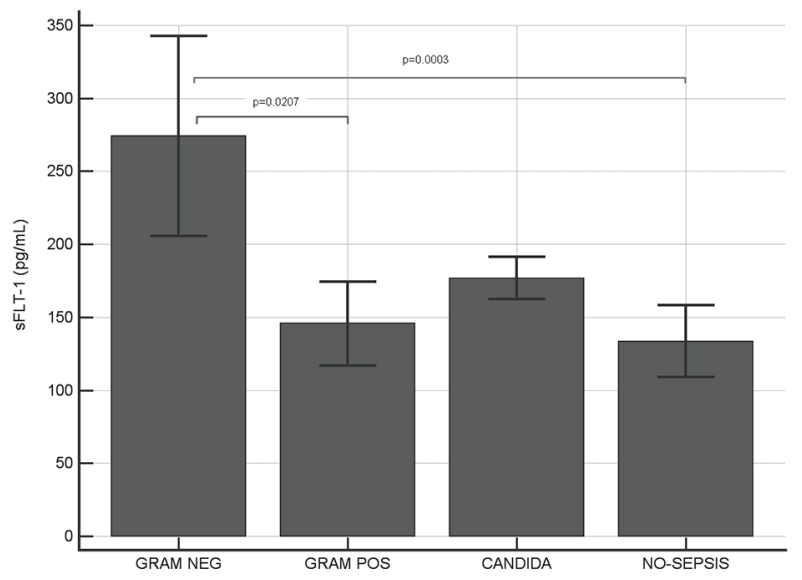

Figure 2. Serum levels of sFlt-1 in Gram-negative, Gram-positive, Candida infections and in sepsis-negative individuals. Significant higher levels of sFlt-1 have been observed in Gram-negative infected patients compared to both Gram-positive and sepsis-negative patients ( $P$ values indicated in figure; where not indicated, difference was not statistically significant). cal parameters are currently used to assess organ dysfunction during sepsis and are used to define the SOFA score, whose changes from baseline are index of sepsis severity, according to the new consensus definitions of sepsis and related criteria $[6,19]$. Multiple Candidate biomarkers of endothelial activation/dysfunction have been proposed for use in evaluating functional status of endothelium in infectious diseases. The present work was aimed to evaluate implication of sFlt-1, a known mediator of vascular tone and permeability, in sepsis. In this perspective, we compared septic patients to a population of not-infected critically ill patients in order to figure out whether the changes were specific to sepsis or broader markers of illness state. First, we found a statistically significant difference in serum level of sFlt-1 between the two groups of patients (Fig. 1), and, as expected, both groups showed significant difference with the healthy control group of not hospitalized individuals. At the same time, we excluded from the no-sepsis control group patients with plausible vascular alteration and not stable conditions such as patients after surgery as well as the newborns. Average and median values of sFlt-1 measured in infected patients $(277.7$ and $165.5 \mathrm{pg} / \mathrm{mL}$, respectively, Table 2) were similar to those previously reported [9-17]. Gram-negative infected individuals $(68 \%$, Table 1) showed higher values of serum sFlt-1 than patients with Gram-positive infections (Fig. 2). As previously published, LPS from Gram-negative bacteria membrane stimulates the macrophages inflammatory response by activation of Tolllike receptor 4 (TLR4), a LPS receptor on the cytoplasmic membrane. This has been proposed to orchestrate immune responses during gram-negative bacterial infection and to be as- 

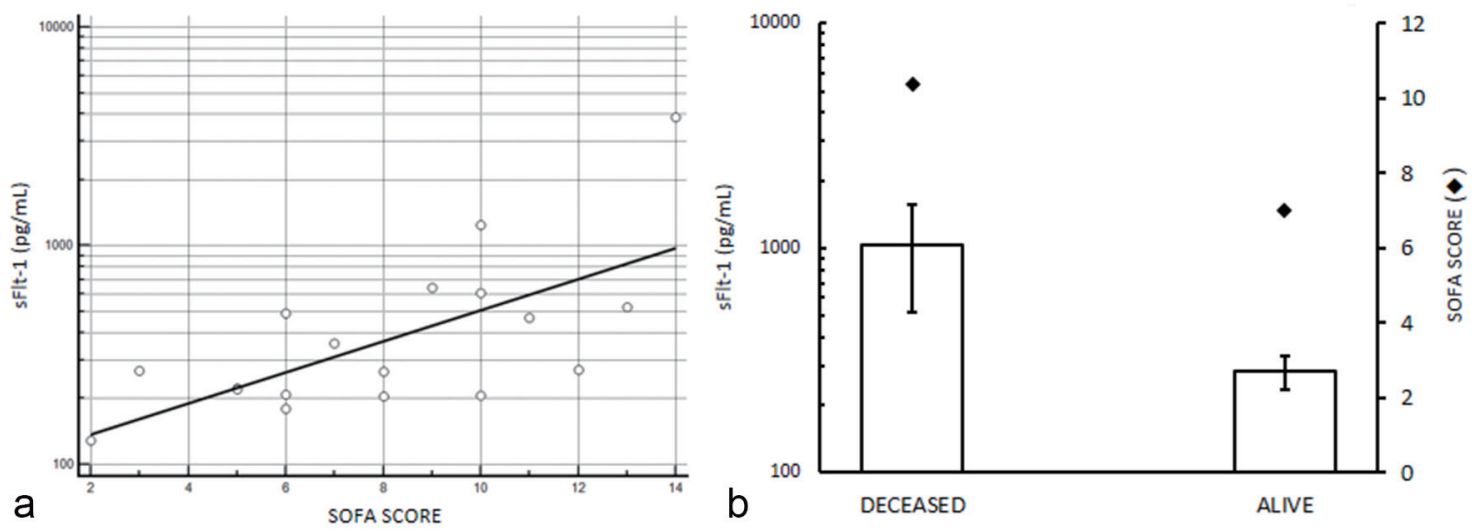

Figure 3. sFIt-1 and sequential organ failure assessment in selected septic patients with serum levels of sFIt-1 higher than 190 $\mathrm{pg} / \mathrm{mL}$ (see text for details). (a) sFlt-1 and SOFA score correlation, $\mathrm{P}<0.0001$ by Chi-squared test for trend. (b) serum levels of sFlt-1 mean values in patients deceased after sepsis and in alive individuals; SOFA score mean values are reported in the second axis. Higher serum levels of sFIt-1 and SOFA score are observed in deceased patients compared to survived $(P<0.05$ by Chi-squared test). sFIt-1 values are showed in semilogarithmic plot.

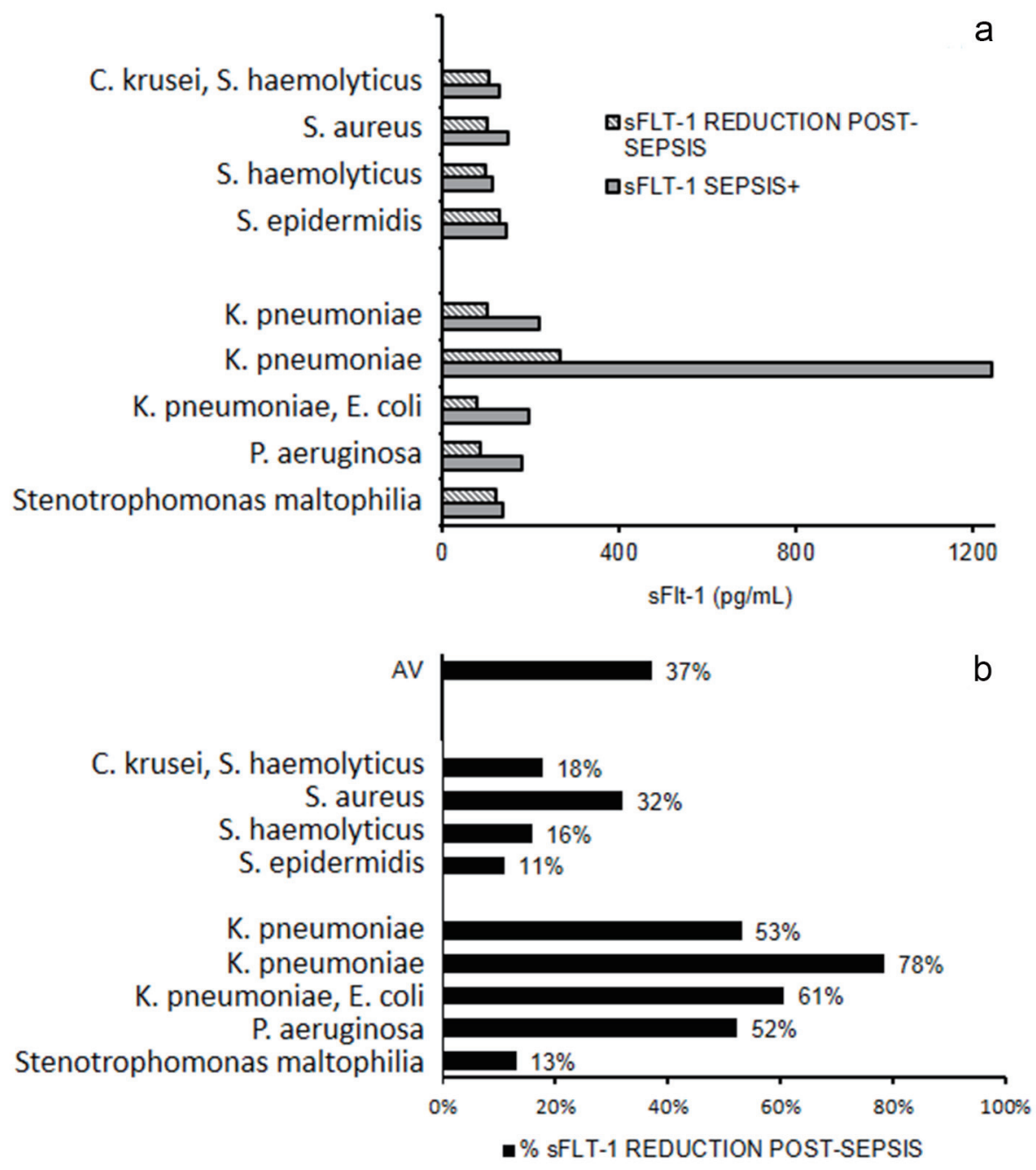

Figure 4. sFlt-1 after sepsis resolution. (a) Serum levels of sFlt-1 measured in nine patients during and after sepsis. (b) Percentage reduction of sFlt-1 after sepsis. AV: mean percentage reduction. 
sociated to septic shock by excessive LPS challenge [20, 21].

Several markers of endothelial cell activation have been associated to sequential organ failure in septic patients, including sFlt-1 [22] and angiopoietin/Tie-2 axis as potential marker of sepsis diagnosis and outcome [6, 23-26], although no biomarker has achieved a uniform reproducibility across patients' population and capacity to identify patients at high risk for complications or mortality, and none of them respond to criteria for ideal biomarker [7]. In the present study, we found a statistically significant positive trend of correlation $(\mathrm{P}<0.0001)$ between the increased levels of sFlt-1 (mean: $528.8 \mathrm{pg} / \mathrm{mL}$, in selected 23 patients with serum level over the upper limit of median 95\% CI) and sequential organ failure evaluated according to the SOFA score (average: 8 ). Moreover, sFlt-1 and SOFA score were significantly higher in not-survivor individuals $(8$ of 20$)$ than survivors $(P<0.05$ by Chi-squared test). After resolution of sepsis and negativization of blood culture, we found an average $37 \%$ decrease of serum levels of sFlt-1; the highest variation of pre- and postsepsis was due to Gram-negative infection (i.e., Klebsiella pneumoniae and Pseudomonas aeruginosa). We did not find any marked decrease in sFlt-1 concentration in no-sepsis control when the assay was repeated after a time interval similar to that of blood culture negativization. On the basis of our results, changes in sFlt-1 serum levels appear associated to the endothelial dysfunction of septic patients. The biological mechanism by which sFlt-1 is involved in regulating pathophysiology of sepsis is, so far, not completely understood. In this process, it is known that the sFlt-1 ligand, VEGF, is an important mediator of endothelial activation by intracellular signaling for the release of cytokines and chemokines, for the expression of procoagulant molecule, the increase of permeability and induction of cell adhesion molecules. However, the soluble receptor, sFlt- 1 , acts differently than membranebound receptors because it does not bring the VEGF signal inside the cell, competing with membrane bound Flt-1 for binding VEGF and thus acting as a decoy molecule. It has been proposed that sFlt-1 could contribute to the systemic anti-inflammatory host response to infection [27]. Our data showed a compensatory elevation of sFlt-1 level in serum of infected patients. The question of whether several fold higher elevation of sFlt-1 could effectively block the endothelial barrier disruption during sepsis is an exciting open question that remains to be answered.

In conclusion, although the limitation due to the small size of studied population, our results highlight the association of endothelial damage with the presence of the infection and the possibility to use serum levels of sFlt-1 as a maker of it. Further studies in a broader population as well as a larger correlation with organ failure assessment and LPS involvement to predict worst outcomes of the patients are ongoing to support present results.

\section{Conflict of Interest}

All authors declare no conflict of interest with any financial organization regarding the items discussed in the manuscript.

\section{References}

1. Aird WC. The role of the endothelium in severe sepsis and multiple organ dysfunction syndrome. Blood. 2003;101(10):3765-3777.

2. De Backer D, Orbegozo Cortes D, Donadello K, Vincent JL. Pathophysiology of microcirculatory dysfunction and the pathogenesis of septic shock. Virulence. 2014;5(1):7379.

3. Martinod K, Wagner DD. Thrombosis: tangled up in NETs. Blood. 2014;123(18):2768-2776.

4. Meziani F, Delabranche X, Asfar P, Toti F. Bench-to-bedside review: circulating microparticles - a new player in sepsis? Crit Care. 2010;14(5):236.

5. Engelmann B, Massberg S. Thrombosis as an intravascular effector of innate immunity. Nat Rev Immunol. 2013;13(1):34-45.

6. Gotts JE, Matthay MA. Sepsis: pathophysiology and clinical management. BMJ. 2016;353:i1585.

7. Page AV, Liles WC. Biomarkers of endothelial activation/dysfunction in infectious diseases. Virulence. 2013;4(6):507-516.

8. Ferrara N, Gerber HP, LeCouter J. The biology of VEGF and its receptors. Nat Med. 2003;9(6):669-676.

9. Pickkers P, Sprong T, Eijk L, Hoeven H, Smits P, Deuren M. Vascular endothelial growth factor is increased during the first 48 hours of human septic shock and correlates with vascular permeability. Shock. 2005;24(6):508-512.

10. Shapiro NI, Yano K, Okada H, Fischer C, Howell M, Spokes KC, Ngo L, et al. A prospective, observational study of soluble FLT-1 and vascular endothelial growth factor in sepsis. Shock. 2008;29(4):452-457.

11. Alves BE, Montalvao SA, Aranha FJ, Lorand-Metze I, De Souza CA, Annichino-Bizzacchi JM, De Paula EV. Time-course of sFlt-1 and VEGF-A release in neutropenic patients with sepsis and septic shock: a prospective study. J Transl Med. 2011;9:23.

12. van der Flier M, van Leeuwen HJ, van Kessel KP, Kimpen JL, Hoepelman AI, Geelen SP. Plasma vascular endothelial growth factor in severe sepsis. Shock. 2005;23(1):3538.

13. Karlsson S, Pettila V, Tenhunen J, Lund V, Hovilehto S, Ruokonen E, Finnsepsis Study G. Vascular endothelial growth factor in severe sepsis and septic shock. Anesth Analg. 2008;106(6):1820-1826.

14. Yang KY, Liu KT, Chen YC, Chen CS, Lee YC, Perng RP, Feng JY. Plasma soluble vascular endothelial growth factor receptor-1 levels predict outcomes of pneumoniarelated septic shock patients: a prospective observational study. Crit Care. 2011;15(1):R11.

15. Maroeska Te Loo D, Bosma N, Van Hinsbergh V, Span P, De Waal R, Clarijs R, Sweep C, et al. Elevated levels of vascular endothelial growth factor in serum of patients with D+ HUS. Pediatr Nephrol. 2004;19(7):754-760.

16. Conroy AL, Liles WC, Molyneux ME, Rogerson SJ, Kain KC. Performance characteristics of combinations of host biomarkers to identify women with occult placental malaria: a case-control study from Malawi. PLoS One. 
2011;6(12):e28540.

17. Seet RC, Chow AW, Quek AM, Chan YH, Lim EC. Relationship between circulating vascular endothelial growth factor and its soluble receptors in adults with dengue virus infection: a case-control study. Int J Infect Dis. 2009;13(5):e248-253.

18. Zeisler H, Llurba E, Chantraine F, Vatish M, Staff AC, Sennstrom M, Olovsson M, et al. Predictive value of the sFlt-1:PIGF ratio in women with suspected preeclampsia. N Engl J Med. 2016;374(1):13-22.

19. Singer M, Deutschman CS, Seymour CW, Shankar-Hari M, Annane D, Bauer M, Bellomo R, et al. The third international consensus definitions for sepsis and septic shock (Sepsis-3). JAMA. 2016;315(8):801-810.

20. Gu L, Meng R, Tang Y, Zhao K, Liang F, Zhang R, Xue $\mathrm{Q}$, et al. Toll like receptor 4 signaling licenses the cytosolic transport of lipopolysaccharide from bacterial outer membrane vesicles. Shock. 2018.

21. Yi YS. Caspase-11 non-canonical inflammasome: a critical sensor of intracellular lipopolysaccharide in macrophage-mediated inflammatory responses. Immunology. 2017;152(2):207-217.

22. Shapiro NI, Schuetz P, Yano K, Sorasaki M, Parikh SM, Jones AE, Trzeciak S, et al. The association of endothelial cell signaling, severity of illness, and organ dysfunction in sepsis. Crit Care. 2010;14(5):R182.

23. Giuliano JS, Jr., Lahni PM, Harmon K, Wong HR, Doughty LA, Carcillo JA, Zingarelli B, et al. Admission angiopoietin levels in children with septic shock. Shock. 2007;28(6):650-654.

24. Kumpers P, Lukasz A, David S, Horn R, Hafer C, Faulhaber-Walter R, Fliser D, et al. Excess circulating angiopoietin-2 is a strong predictor of mortality in critically ill medical patients. Crit Care. 2008;12(6):R147.

25. Siner JM, Bhandari V, Engle KM, Elias JA, Siegel MD. Elevated serum angiopoietin 2 levels are associated with increased mortality in sepsis. Shock. 2009;31(4):348353.

26. Alves BE, Montalvao SA, Aranha FJ, Siegl TF, Souza CA, Lorand-Metze I, Annichino-Bizzacchi JM, et al. Imbalances in serum angiopoietin concentrations are early predictors of septic shock development in patients with post chemotherapy febrile neutropenia. BMC Infect Dis. 2010;10:143.

27. Tsao PN, Chan FT, Wei SC, Hsieh WS, Chou HC, Su YN, Chen CY, et al. Soluble vascular endothelial growth factor receptor-1 protects mice in sepsis. Crit Care Med. 2007;35(8):1955-1960. 